\title{
LA FECUNDIDAD ENTRE LOS MEXICANO- AMERICANOS EN RELACIÓN A LOS CAMBIANTES PATRONES REPRODUCTIVOS EN MÉXICO Y LOS ESTADOS UNIDOS
}

\author{
FRANCISCO ALBA \\ El Colegio de México
}

\section{Introducción}

A MANERA DE NOTA introductoria se presentan algunas breves observaciones sobre los movimientos de población mexicana hacia los Estados Unidos que han dado origen a la existencia de una minoría mexicanoamericana en ese país, sin olvidar que estos movimientos se dan en un contexto de relaciones que encierran vínculos culturales e históricos.

Hacia mediados del siglo XIX, México había perdido las provincias septentrionales en favor de los Estados Unidos (las provincias del norte equivalían aproximadamente a la mitad del territorio original del país al momento de la independencia). Al norte de la nueva línea fronteriza, que a partir de ese momento dividió a los dos países, quedó una población de ascendencia novohispana y mexicana çuyo desarrollo se ubicaba fuera de la corriente principal de la población estadounidense. En las postrimerías del siglo XIX volverían a intensificarse las relaciones entre las dos economías involucrando una intensificación de movimientos temporales. y de variados asentamientos de población mexicana hacia los Estados Unidos.

Estos movimientos de población mexicana y su correlativo patrón de asentamiento principalmente a todo lo ancho del suroeste norteamericano son fenómenos ya centenarios que forman parte de una tradición y una relación permanente entre los dos países. No obstante

\footnotetext{
*Texto ligeramente modificado de una comunicación preparada para la sesión sobre "The Demographic Behavior of the Same Ethnic Groups in Different Countries". Conferencia General de la IUSSP, Manila, Filipinas, 9-16 de diciembre, 1981. Agradezco a Joseph E. Potter señalamientos y comentarios hechos a una versión anterior; ello no involucra su responsabilidad.
} 
lo anterior, el fenómeno de los mexicanos en Estados Unidos se encuentra relativamente poco documentado. Varias razones explican esta situación. En un primer momento, tal vez hasta bien avanzados los años 20, la línea divisoria no separaba ni impedía drásticamente la libre movilidad de la población entre los dos lados de la frontera. Los movimientos migratorios de la población mexicana hacia el país del norte ha tenido y continúa teniendo un claro y predominante rasgo estacional o temporal, a la vez que el patrón migratorio muestra un carácter repetitivo y rotatorio. Es asi que mientras los flujos anuales comprenden a varios cientos de miles de personas, los incrementos netos a la población de origen mexicano en los Estados Unidos son mucho menores. Finalmente el hecho mismo de lo indocumentado de los cruces, en uno y otro sentido, dificulta los esfuerzos de estimación cuantitativa tanto de los movimientos y de la población "flotante" de origen mexicano en los Estados Unidos como del número mismo de mexicano-americanos.

Resultados preliminares del censo de los EE.UU. de 1980 indican que más de ocho millones de personas de origen o descendencia mexicana residen en dicho país. Esta información contrasta fuertemente con la del censo de 1970; de cuatro y medio millones de descendencia mexicana. En ese año, 1970, la gran mayoría (casi 90 porciento) vivía en cinco estados del suroeste norteamericano -California, Texas, Nuevo México, Arizona y Colorado- en los que a la vez las poblaciones de origen mexicano o hispano constituyen el grupo minoritario más numeroso. En California y Texas tan sólo estaban contenidas las tres cuartas partes de esta población. ${ }^{1}$

En sintesis, la actual población de origen mexicano en los Estados Unidos es el producto mayoritariamente de los movimientos migratorios del presente siglo, si bien muchos mexicanos llegaron y se asentaron en aquel país desde fines del siglo pasado, o incluso más atrás en el tiempo. Puesto que población mexicana y novohispana, ha residido en el actual territorio de los Estados Unidos por lapsos mucho más extensos existen números susțanciales de población mexicanoamericana que son nativos de los Estados Unidos y de padres también nativos.

¿Cómo se ubica el grupo de mexicano-americanos respecto de la población total o mayoritaria de los Estados Unidos? Los mexicanoamericanos muestran una situación socioeconómica de desventaja

1 Cuatro de estos cinco estados son fronterizos con México. Un grupo importante de población de origen mexicano reside en el Estado de lllinois. Las estadísticas sobre la población de origen mexicano se derivan de autoidentificación, como ser de origen o descendencia mexicana, o de una aproximación a partir de las poblaciones de apellido "español" que residen en los cinco estados del suroeste mencionados. 
reflejada en más bajos niveles educativos y ocupacionales y un menor nivel de ingresos. Los mexicano-americanos ocupaban hasta $1970 \mathrm{em}$ pleos de baja categoría, no obstante la considerable movilidad social ascendente experimentada durante varios de los decenios anteriores. Los mexicano-americanos se encuentran entre los segmentos más pobres de la sociedad estadounidense. El ingreso familiar de la población de descendencia mexicana promediaba $\$ 6.960 .00$ dólares en comparación con $\$ 10,100.00$ de la población blanca de origen no-hispano. ${ }^{2}$ La situación ha ido mejorando, pero el progreso parece lento.

En relación con los migrantes mexicanos, sobre todo los que caen en la categoría de indocumentados, éstos suelen exhibir, en general, una calificación y "status" ocupacional inferior que el de los migrantes que ingresan a los Estados Unidos en forma documentada. Sin embargo un creciente número de migrantes mexicanos se podrían ubicar también en posiciones intermedias de las escalas ocupaciona1es. ${ }^{3}$ Un indicador conciso de la situación presente viene dado por la repetida constatación de que la población mexicana en los Estados Unidos, sean residentes legales o migrantes indocumentados, se encuentra empleada de manera consistente en los llamados "mercados laborales secundarios".

En el marco de una discusión en torno al comportamiento demográfico de un mismo grupo étnico (una nacionalidad) en contextos (países) diferentes se eligió dirigir la atención a la cuestión del comportamiento comparativo de la fecundidad entre las poblaciones mexicano-americana, mexicana y norteamericana.

¿Cuál es la importancia de un tema como éste? México se ha caracterizado por haber mantenido por largos períodos una muy elevada fecundidad. En un período tan reciente como el que va de 1960 a 1974 la tasa global de fecundidad para el país en su conjunto se estima entre 6.5 y 6.8 ; nivel que se sitúa muy significativamente por encima de las tasas que prevalecían en la misma época para los Estados Unidos cuando decrecieron del 3.6 en 1960 al 2.0 en $1972 .{ }^{4}$ Parece pertinente, en consecuencia, examinar si la población de origen o descendencia mexicana ha adaptado su fecundidad de acuerdo a la experiencia de vivir en un país caracterizado por un nivel de fecundidad

\footnotetext{
2 Otros grupos de descendencia "hispana" son los puertorriqueños, hispanos, cubanos y descendientes de países centro y sudamericanos.

3 Francisco Alba, "Exodo silencioso: Ja migración de trabajadores mexicanos a Estados Unidos." Foro Internacional, octubre, 1976, pp. 152-179.

4 Dirección General de Estadística, Los niveles de Fecundidad en México 1960-1974. Estadísticas Vitales. Serie 1, No. 3. Secretaría de Programación y Presupuesto, México, Junio, 1978; The Population of the United States of America, C.I.C.R.E.D. Series, 1974.
} 
muy inferior al prevaleciente en su país de origen o si ha mantenido un comportamiento similar al prevaleciente en este último país. Incidentalmente hay que notar que la población mexicana recientemente ha estado pasando por cambios pronunciados en su fecundidad. La tasa global de fecundidad ha caido en un 25 por ciento en menos de una década, de un valor estimado de 6.64 para el período 19711973 a uno de 4.94 para $1977-1979.5$

Las consideraciones que siguen podrían ubicarse en el contex to de un debate que tiende a girar en torno a la contribución de los valores estructurales y culturales en la explicación del comportamiento demográfico. Este parece ser el trasfondo de mucha de la literatura que trata sobre este tema. Los resultados de la revisión de la literatura revisada se reportan, primero, en términos de un análisis comparativo de la fecundidad sobre diferencias o similitudes de niveles entre las poblaciones contempladas y, segundo, sobre diferencias o similitudes en patrones de variación de la fecundidad según situación socioeconómica. Se cierra con una breve discusión.

\section{Comportamiento comparativo de la fecundidad}

\section{i) Niveles y tendencias}

En general, la fecundidad de la población mexicano-americana es sistemáticamente más elevada que la de la población norteamericana en su conjunto y se asemeja a la de la población mexicana. Sin embargo, las comparaciones no pueden ser directas. Los instrumentos de medición no suelen ser uniformes y los indicadores utilizados para aprehender las fuerzas y factores sociales en operación no pueden ser unívocamente comparables.

Las precisiones anteriores deben tenerse muy presentes en las comparaciones siguientes. Con relación a la población de origen mexicano residiendo en los Estados Unidos y a la población mexicana (en México) la situación en 1970 se podría resumir brevemente. E1 cuadro 1 contiene información sobre el número promedio de hijos nacidos de mujeres entre los 35 y 44 años de edad para cuatro grupos: i) mujeres nacidas en México viviendo en Estados Unidos que no han completado la educación pre-universitaria (menos de 12 años de escuela); ii) mujeres de origen mexicano (tanto nacidas en México como en Estados Unidos) viviendo en Estados Unidos que no han

5 Fecundidad y uso de métodos anticonceptivos en México, Instituto Mexicano del Seguro Social, 1981. 
completado la educación elemental (menos de 8 años de escuela); iii) mujeres mexicanas viviendo en zonas urbanas (localidades de 2500 habitantes o más); y iv) mujeres mexicanas viviendo en zonas rurales. Los grupos que parecen ser los más comparables son el ii) y el iii): por un lado la población de origen mexicano en los Estados Unidos es predominantemente urbana, por otro la gran mayoría de las mujeres mexicanas no han completado la educación primaria. Para las mujeres en el mercado de trabajo las diferencias no parecen significativas: 4.63 hijos en promedio entre las residentes en Estados Unidos contra 4.75 entre las residentes en México. Para las mujeres fuera del mercado de trabajo la diferencia es ligera: 6.12 contra 5.53 respectivamente.

Cuadro 1

Número medio de hijos nacidos de mujeres casadas en edades entre 35 y 44 , segon empleo, educación y residencia rural o urbana. poblactón de origen mexicano en Estados unidos y México, 1970

\begin{tabular}{|c|c|c|c|c|}
\hline & \multicolumn{2}{|c|}{$\begin{array}{l}\text { Poblacion de origen mextcano } \\
\text { en los Estados Unidos }\end{array}$} & \multicolumn{2}{|c|}{ Poblacion mexicana } \\
\hline & $\begin{array}{c}\text { Nacida en México } \\
\text { menos de } 12 \text { años } \\
\text { de escuela }\end{array}$ & $\begin{array}{l}\text { De origen mexicano } \\
\text { menos de } 8 \text { años } \\
\text { de escuela }\end{array}$ & Urbana & Rural \\
\hline Empleado & 4. 31 & 4.63 & 4.75 & 5.41 \\
\hline No empleado & 5.16 & 5.53 & 6.12 & 6.91 \\
\hline
\end{tabular}

La imagen que se desprende de comparar la fecundidad de los mexicano-americanos con la fecundidad general de los Estados Unidos es el de una fecundidad sustancialmente más elevada entre la población de origen mexicano que la que prevalece entre la población en general. El número promedio de hijos de las mujeres entre 35 y 44 años de edad de origen mexicano era en 1969 de 4.1, comparado con 3.0 para las mujeres de todas las etnias; esto es, la fecundidad de la población mexicano-americana superaba en 47 por ciento la fecundidad general de la población norteamericana (ver cuadro 2). La mayor fecundidad de las mujeres mexicano-americanas se manifiesta en todos los grupos de edad. ${ }^{6}$

6 U.S. Department of Commerce. Bureau of the Census, Current Population Reports, Serie P-20, No. 226, Fertility Variations by Ethnic Origin, noviembre, 1971. 
Cuadro 2

\begin{tabular}{|c|c|c|c|c|c|}
\hline \multirow{2}{*}{ Raza origen } & \multicolumn{5}{|c|}{ Hijos nacidos vivos } \\
\hline & por 1 & 1000 & mujeres & $\begin{array}{l}\text { por } 1 \\
\text { alguna }\end{array}$ & $\begin{array}{l}1000 \text { mujeres } \\
\text { a vez casadas }\end{array}$ \\
\hline Todas & & & 003 & & 3143 \\
\hline Blanca & & 29 & 923 & & 3054 \\
\hline Negra & & 36 & 649 & $\cdot$ & 3869 \\
\hline Mexicana & & 4 & 429 & & 4619 \\
\hline
\end{tabular}

Fuente: U.S. Bureau of the Census, Fertility Variations by Ethnic Origin, noviembre, 1971 .

Si bien la fecundidad de la población mexicano-americana ha sido históricamente elevada y aún persisten amplios diferenciales de fecundidad entre las dos poblaciones - la de origen mexicano y el resto de la población blanca- las tendencias en la fecundidad sugieren una reducción de los diferenciales durante las últimas dos o tres décadas. El diferencial entre las mujeres comprendidas entre las edades 35-44 años excedía el 100 por ciento en 1950 y se redujo al 45 por ciento en 1970 (ver cuadro 3).

Evidencia colateral tiende a confirmar que la fecundidad entre la población mexicano-americana ha estado declinando. Hasta 1960 no aparecían señales de reducción entre las diversas generaciones: las mujeres mexicano-americanas entre 35 y 44 años de edad nacidas en México tenían 3628 hijos por cada mil mujeres en comparación con los 3775 hijos por cada mil mujeres mexicano-americanas nacidas en los Estados Unidos y de padres también nacidos en dicho país. ${ }^{7} \mathrm{Sin}$ embargo en 1970 surge un patrón de reducción de la fecuñdidad según generaciones; se ha encontrado que las generaciones de orden tercero o más alto (mujeres mexicano-americanas nacidas en los Estados Unidos de padres nacidos también ahí) tienen consistentemente menos hijos que las generaciones de orden primero o segundo (ver cuadro 4). Si bien las generaciones más antiguas tenían menos hijos a edades más jóvenes que las generaciones más recientes ello no significa, sin embargo, que eventualmente ambas generaciones terminen teniendo al final del período reproductivo un número comparable de nacimientos. ${ }^{8}$

7 Peter Uhlenberg, "Fertility Patterns within the Mexican-American Population", Social Biology, marzo, 1973, pp. 30-39.

8 lirank D. Bean et al., "Patterns of Fertility Variation Among Mexican Immigrants to the United States", Documento No. 2.016, U.T., Austin, Texas Population Research Center, Serie 2: 1979-1980. 
Cuadro 3

Hijos nacidos por 1000 mujeres, reportadas y ajustadas por edad, según etnia

o apellido, y grupos de edad: cinco estados del suroeste, 1950-1970.

\begin{tabular}{|c|c|c|c|c|c|c|c|c|c|c|c|c|}
\hline \multirow[b]{2}{*}{ Edad } & \multicolumn{3}{|c|}{$1970^{a}$} & \multicolumn{3}{|c|}{$1969 b$} & \multicolumn{3}{|c|}{$1960 \mathrm{c}$} & \multicolumn{3}{|c|}{$1950 d$} \\
\hline & $\begin{array}{l}\text { Idiowa } \\
\text { apellido } \\
\text { español }\end{array}$ & $\begin{array}{c}\text { Blancos } \\
\text { no } \\
\text { hispanos }\end{array}$ & $\begin{array}{l}\text { Diferencia } \\
\text { porcencual }\end{array}$ & $\begin{array}{c}\text { Origen } \\
\text { Mexicano }\end{array}$ & $\begin{array}{l}\text { Blancos } \\
\text { otro } \\
\text { origen }\end{array}$ & $\begin{array}{l}\text { Diferencia } \\
\text { porcentual }\end{array}$ & $\begin{array}{l}\text { Apellido } \\
\text { español } \\
\text { (blancos) }\end{array}$ & $\begin{array}{c}\text { otro } \\
\text { apellido } \\
\text { (blancos) }\end{array}$ & $\begin{array}{l}\text { Diferencia } \\
\text { porcentual }\end{array}$ & $\begin{array}{l}\text { Apellido } \\
\text { españo1 } \\
\text { (blancos) }\end{array}$ & $\begin{array}{c}\text { Otro } \\
\text { apellido } \\
\text { (blancos) }\end{array}$ & $\begin{array}{l}\text { Diferencia } \\
\text { porcentual }\end{array}$ \\
\hline $\begin{array}{l}15-44 \\
\text { ajustada }\end{array}$ & $\begin{array}{l}2,004 \\
2,306\end{array}$ & $\begin{array}{l}1,709 \\
1,649\end{array}$ & $\begin{array}{l}17.3 \\
39.8\end{array}$ & $\begin{array}{l}2,119 \\
2,537\end{array}$ & $\begin{array}{l}1,539 \\
1,686\end{array}$ & $\begin{array}{l}37.7 \\
50.6\end{array}$ & $\begin{array}{l}2,290 \\
2,495\end{array}$ & $\begin{array}{l}1,697 \\
1,697\end{array}$ & $\begin{array}{l}34.9 \\
47.0\end{array}$ & $\begin{array}{l}2,046 \\
2,332\end{array}$ & $\begin{array}{l}1,329 \\
1,296\end{array}$ & $\begin{array}{l}53.9 \\
79.9\end{array}$ \\
\hline $\begin{array}{l}15-24 \\
\text { ajustada }\end{array}$ & $\begin{array}{l}498 \\
685\end{array}$ & $\begin{array}{l}334 \\
329\end{array}$ & $\begin{array}{r}49.1 \\
108.2\end{array}$ & $\begin{array}{l}403 \\
456\end{array}$ & $\begin{array}{l}390 \\
363\end{array}$ & $\begin{array}{r}3.3 \\
25.6\end{array}$ & $\begin{array}{l}694 \\
725\end{array}$ & $\begin{array}{l}595 \\
595\end{array}$ & $\begin{array}{l}16.6 \\
21.8\end{array}$ & $\begin{array}{l}635 \\
609\end{array}$ & $\begin{array}{l}491 \\
441\end{array}$ & $\begin{array}{l}29.3 \\
38.1\end{array}$ \\
\hline $\begin{array}{l}25-34 \\
\text { ajustada }\end{array}$ & $\begin{array}{l}2,612 \\
2,636\end{array}$ & $\begin{array}{l}1,953 \\
1,974\end{array}$ & $\begin{array}{l}33.7 \\
33.5\end{array}$ & $\begin{array}{l}2,768 \\
2,816\end{array}$ & $\begin{array}{l}2,016 \\
2,040\end{array}$ & $\begin{array}{l}37.3 \\
38.0\end{array}$ & $\begin{array}{l}2,950 \\
2,950\end{array}$ & $\begin{array}{l}2,151 \\
2,151\end{array}$ & $\begin{array}{l}37.1 \\
37.6\end{array}$ & $\begin{array}{l}2,620 \\
2,661\end{array}$ & $\begin{array}{l}1,565 \\
1,573\end{array}$ & $\begin{array}{l}67.4 \\
69.2\end{array}$ \\
\hline $\begin{array}{l}35-44 \\
\text { ajustada }\end{array}$ & $\begin{array}{l}3,895 \\
3,899\end{array}$ & $\begin{array}{l}2,676 \\
2,687\end{array}$ & $\begin{array}{l}45.6 \\
45.1\end{array}$ & $\begin{array}{l}4,358 \\
4,379\end{array}$ & $\begin{array}{l}2,710 \\
2,718\end{array}$ & $\begin{array}{l}60.8 \\
61.1\end{array}$ & $\begin{array}{l}3,810 \\
3,796\end{array}$ & $\begin{array}{l}2,258 \\
2,258\end{array}$ & $\begin{array}{l}68.7 \\
68.1\end{array}$ & $\begin{array}{l}3,921 \\
3,916\end{array}$ & $\begin{array}{l}1,816 \\
1,817\end{array}$ & $\begin{array}{l}115.9 \\
115.5\end{array}$ \\
\hline
\end{tabular}

Cambios porcentuales en el número de hijos nacidos

\begin{tabular}{|c|c|c|c|c|c|c|c|c|c|c|}
\hline \multirow[b]{2}{*}{ Edad } & \multicolumn{2}{|c|}{$1960-1970$} & \multicolumn{2}{|c|}{$1960-1969$} & \multicolumn{2}{|c|}{$1950-1960$} & \multicolumn{2}{|c|}{$1950-1970$} & \multicolumn{2}{|c|}{$1950-1969$} \\
\hline & $\begin{array}{l}\text { Ydioma o } \\
\text { apellido } \\
\text { español }\end{array}$ & $\begin{array}{l}\text { Otro idioma } \\
\text { o apellido } \\
\text { (blances) }\end{array}$ & $\begin{array}{l}\text { Origen } \\
\text { mexicano } \\
\text { o hispano }\end{array}$ & $\begin{array}{l}\text { Otro apellido } \\
\text { u origen } \\
\text { (blancos) }\end{array}$ & $\begin{array}{l}\text { Apel1ido } \\
\text { español } \\
\text { (blancos) }\end{array}$ & $\begin{array}{c}\text { Otro } \\
\text { apellido } \\
\text { (blancos) }\end{array}$ & $\begin{array}{l}\text { Idioma o } \\
\text { ape11ido } \\
\text { español }\end{array}$ & $\begin{array}{l}\text { Otro idioma } \\
\text { o apellido } \\
\text { (blancos) }\end{array}$ & $\begin{array}{c}\text { Apellido } \\
\text { español u } \\
\text { origen mexicano }\end{array}$ & $\begin{array}{l}\text { Otro apellido } \\
\text { u origen } \\
\text { (blancos) }\end{array}$ \\
\hline $\begin{array}{l}15-44 \\
\text { ajugrada }\end{array}$ & $\begin{array}{r}-12.5 \\
-7.6\end{array}$ & $\begin{array}{r}0.7 \\
-2.8\end{array}$ & $\begin{array}{l}-7.5 \\
+1.7\end{array}$ & $\begin{array}{l}=9.3 \\
=0.7\end{array}$ & $\begin{array}{r}11.9 \\
7.0\end{array}$ & $\begin{array}{l}27.7 \\
30.9\end{array}$ & $\begin{array}{r}-2.1 \\
1.4\end{array}$ & $\begin{array}{l}28.6 \\
27.2\end{array}$ & $\begin{array}{l}3.6 \\
8.8\end{array}$ & $\begin{array}{l}15.8 \\
30.0\end{array}$ \\
\hline $\begin{array}{l}15-24 \\
\text { ajustada }\end{array}$ & $\begin{array}{l}-28.2 \\
-5.5\end{array}$ & $\begin{array}{l}-43.9 \\
-44.7\end{array}$ & $\begin{array}{l}-41.9 \\
-37.1\end{array}$ & $\begin{array}{l}-34.5 \\
-39.0\end{array}$ & $\begin{array}{r}9.3 \\
19.0\end{array}$ & $\begin{array}{l}21.1 \\
34.9\end{array}$ & $\begin{array}{l}-21.6 \\
-12.5\end{array}$ & $\begin{array}{l}-32.0 \\
-25.4\end{array}$ & $\begin{array}{l}-36.5 \\
-25.1\end{array}$ & $\begin{array}{l}-20.6 \\
-17.7\end{array}$ \\
\hline $\begin{array}{l}25-34 \\
\text { ajustada }\end{array}$ & $\begin{array}{l}-11.5 \\
-10.9\end{array}$ & $\begin{array}{l}-9.2 \\
-8.2\end{array}$ & $\begin{array}{l}-6.2 \\
-4.8\end{array}$ & $\begin{array}{r}-6.3 \\
-5.2\end{array}$ & $\begin{array}{l}12.6 \\
11.2\end{array}$ & $\begin{array}{l}37.4 \\
36.7\end{array}$ & $\begin{array}{l}-0.3 \\
-0.0\end{array}$ & $\begin{array}{l}24.8 \\
25.5\end{array}$ & $\begin{array}{l}5.6 \\
5.8\end{array}$ & $\begin{array}{l}28.8 \\
29.7\end{array}$ \\
\hline $\begin{array}{l}35-44 \\
\quad \text { ajus tada }\end{array}$ & $\begin{array}{r}2.2 \\
+2.7\end{array}$ & $\begin{array}{r}18.5 \\
+19.0\end{array}$ & $\begin{array}{r}14.4 \\
+15.4\end{array}$ & $\begin{array}{r}20.0 \\
+20.4\end{array}$ & $\begin{array}{l}-2.8 \\
-3.1\end{array}$ & $\begin{array}{l}24.3 \\
24.3\end{array}$ & $\begin{array}{l}-0.7 \\
-0.4\end{array}$ & $\begin{array}{l}47.4 \\
47.9\end{array}$ & $\begin{array}{l}11.1 \\
\text { i1.. }\end{array}$ & $\begin{array}{l}49.6 \\
49.0\end{array}$ \\
\hline
\end{tabular}

a U.S. Bureau of the Census, United States Census of Dopulation: 1970, General Social and Economic Characteristics, Final Reports PC (1)-C, 1972.

b Unpublished data from the November 1969 Current population Survey supplied trough the coartesy of the Population Division, U.S. Bureau of the Census.

c U.S. Bureau of the Census, United States Census of Population: 1960, Subject Reports, Women by Number of Children Ever Born, Final Report PC (2)-3A, 1964

d U.S. Bureau of the Census, U.S. Census of Population: 1950, Vol. IV, Special Reports, Part 5, Ch. C., Fertility, 1955.

Reproducido de: B.S, Bradshaw and F.D. Bear, "Trends in the Fertílity of Mexican Americans", 1973, pp. 693 y 695 
Cuadro 4

Numero medio de hijos menores de 15 años y menores de 3 años de mujeres

alguna vez casadas en edades de 20 a 34 años, desviaciones estandar,

segtin grupo êtnico $y$ orden generacional: Estados Unidos, 1970

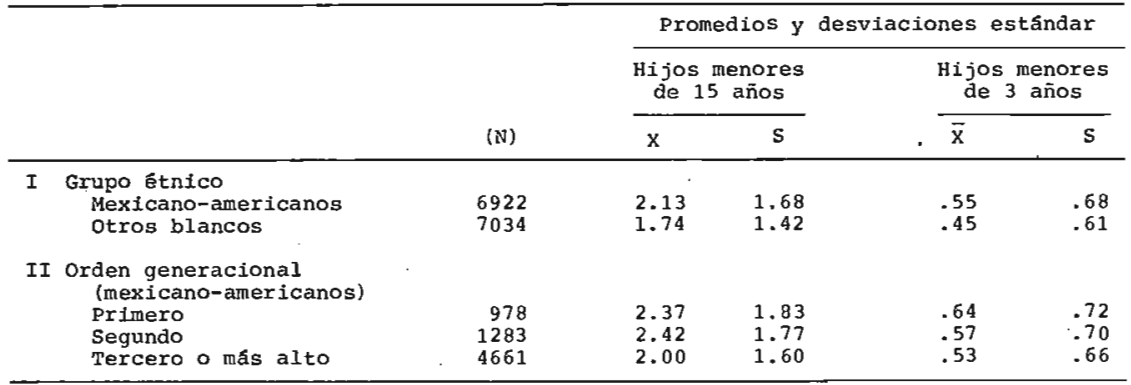

Fuente: 1970 U.S. Public Use Sample.

Reproductdo de: Frank D. Bean et al., "Patterns of Fertility Variation Among Mexican Inmigrants", 1980 , p. 25.

Diversos estudios muestran que para las décadas más recientes los cambios en la fecundidad de la población mexicano-americana han ido paralelos a los patrones de la fecundidad de la población norteamericana; incrementos entre 1950 y 1960 y reducciones entre 1960 y 1970. El cuadro 3 ofrece evidencias al respecto, lo que sugiere que la fecundidad de la población mexicano-americana responde al mismo tipo de factores que al que responden otros grupos (blancos) en los Estados Unidos. ${ }^{9}$ En el mismo cuadro 3 también aparece que las fluctuaciones en la fecundidad de los mexicano-americanos no han sido tan pronunciadas como las de los anglos. De lo anterior surge la cuestión de la medida en que las diferencias en fecundidad hasta aquí observadas entre las dos poblaciones, mexicano-americana y anglo, son producto de diferencias en condiciones socioeconómicas y culturales. ¿Cuál es el peso de la orientación cultural? ¿Actúan las condiciones sociales y económicas como parámetros de referencia del comportamiento demográfico? En la siguiente sección se plantea un acercamiento a estas cuestiones. Los patrones de fecundidad de la población mexicano-americana se comparan, primero, con los patrones de otras poblaciones de los Estados Unidos (los blancos) y, en segundo término, con los patrones de la población de México.

9 Benjamin S. Bradshaw y Frank. D. Bean, "Trends in the Fertility of Mexican-Americans: $1950 ; 1970$ ", Social Science Quarterly, marzo, 1973, pp. 688-696. 


\section{ii) Patrones de comportamiento según condición socioeconómica}

La información disponible sugiere que la fecundidad de la población mexicano-americana varía en relación a la condición socioeconómica en aproximadamente la misma forma a como lo hace la fecundidad de la población blanca total norteamericana (ver cuadro 5). ${ }^{10}$ Existe una tendencia de asociación negativa entre nivel de fecundidad y posición económica y social, utilizando la educación como indicador. (Se considera que el nivel educativo encierra adecuadamente la influencia sobre la fecundidad de un conjunto de factores sociales y económicos). Completar la escuela elemental ( 8 años) o la escuela intermedia (high school: 12 años) actúan como puntos de quiebre.

Cuadro 5

Hijos nacidos por 1000 mujeres entre 15 y 44 años de edad según años de educación para el total de población blanca y población de origen mexicano: Estados Unidos, 1969

\begin{tabular}{lcc}
\hline Años completos de educación & Población blanca & Origen mexicano \\
\hline Elemental & 2,396 & 3,274 \\
menos de 8 años & 2,596 & 3,646 \\
8 años & 2,222 & 2,515 \\
Superior y Universidad & 1,560 & 1,542 \\
Superior & 1,644 & 1,563 \\
1-3 años & 1,393 & 1,616 \\
Unios & 1,794 & 1,497 \\
Tniversidad & 1,292 & $($ a) \\
& 1,634 & 2,179 \\
\hline
\end{tabular}

Fuente: U.S. Bureau of the Census, Current Population Reports, Series P-20, No. 226, Fertility Variations by Ethnic Origin: November 1969, 1971. a: menos de 75000 casos.

Reproducido de: B.S. Bradshaw and F.D. Bean, "Some Aspects of the Fertility of Mexican Americans", 1972, p. 147.

Estudios recientes han encontrado que una parte sustancial de las diferencias en fecundidad entre la población blanca y la mexicanoamericana se asocia con diferencias entre grupos según niveles de educación. Los diferenciales en fecundidad entre ambas poblaciones disminuyen en más de un cincuenta por ciento cuando la educación se mantiene como constante. ${ }^{11}$ El cuadro 6 muestra que una mayor

10 Benjamin S. Bradshaw y Frank D. Bean, "Some Aspects of the Fertility of Mexican Americans". Commission on Population Growth and the American Future. Demographic and Social Aspects of Population Growth, 1972, pp. 143-164.

11 Frank D. Bean et al., "Patterns of Fertility Variation Among Mexican Immigrants", 1980. 
educación se asocia con una reducción de la fecundidad tanto entre los mexicano-americanos como entre los otros blancos norteamericanos. Los datos sugieren que la fecundidad es también entre los mexicano-americanos una respuesta de adaptación al entorno social dentro del que las poblaciones se desenvuelven (no una pura respuesta de tipo prescriptivo exclusivamente debida a factores culturales inmutables).

Cuadro 6

Numero promedio de hijos menores de 15 años de mujeres en edades 20-34 años segôn educación, grupo étnico $y$ orden generacional: Estados Unidos, 1970

\begin{tabular}{|c|c|c|c|c|c|c|}
\hline \multirow{2}{*}{ Grupo étnico } & \multicolumn{6}{|c|}{ Educación } \\
\hline & $0-8$ & $9-11$ & 12 & $13-15$ & $16+$ & Total \\
\hline $\begin{array}{l}\text { Blancos (otros) } \\
\text { MexicanomAmericanos }\end{array}$ & $\begin{array}{l}2.23 \\
2.75\end{array}$ & $\begin{array}{l}2.25 \\
2.51\end{array}$ & $\begin{array}{l}1.77 \\
1.83\end{array}$ & $\begin{array}{l}1.53 \\
1.45\end{array}$ & $\begin{array}{l}1.03 \\
1.02\end{array}$ & $\begin{array}{l}1.74 \\
2.13\end{array}$ \\
\hline Orden generacional & & & & & & . \\
\hline $\begin{array}{l}\text { Primera } \\
\text { Segunda } \\
\text { Tercera o mas alta }\end{array}$ & $\begin{array}{l}2.66 \\
2.87 \\
2.78\end{array}$ & $\begin{array}{l}2.19 \\
2.94 \\
2.42\end{array}$ & $\begin{array}{l}1.93 \\
1.96 \\
1.79\end{array}$ & $\begin{array}{l}1.62 \\
1.79 \\
1.37\end{array}$ & $\begin{array}{r}* \\
1.55 \\
.98\end{array}$ & $\begin{array}{l}2.37 \\
2.42 \\
2.00\end{array}$ \\
\hline
\end{tabular}

Numero promedio de hijos menores de 15 años de mujeres en edades 20-34 años segín educacion, grupo étnico y orden generacional: Estados Unidos, 1976

\begin{tabular}{|c|c|c|c|c|c|c|}
\hline \multirow{2}{*}{ Grupe étnico } & \multicolumn{6}{|c|}{ Educación } \\
\hline & $0-8$ & $9-11$ & 12 & $13-15$ & $16+$ & Total \\
\hline $\begin{array}{l}\text { Blancos (otros) } \\
\text { Mexicano-Americanos }\end{array}$ & $\begin{array}{l}2.22 \\
2.43\end{array}$ & $\begin{array}{l}1.98 \\
2.25\end{array}$ & $\begin{array}{l}1.51 \\
1.61\end{array}$ & $\begin{array}{l}1.26 \\
1.55\end{array}$ & $\begin{array}{l}.93 \\
.88\end{array}$ & $\begin{array}{l}1.43 \\
1.96\end{array}$ \\
\hline \multicolumn{7}{|l|}{ Orden generacional } \\
\hline $\begin{array}{l}\text { Primera } \\
\text { Segunda o más alta }\end{array}$ & $\begin{array}{l}2.26 \\
2.73\end{array}$ & $\begin{array}{l}2.03 \\
2.30\end{array}$ & $\begin{array}{l}2.02 \\
1.52\end{array}$ & 1.59 & .87 & $\begin{array}{l}2.10 \\
1.90\end{array}$ \\
\hline
\end{tabular}

Fuentes: 1970 U.S. Public Use Sample; 1976 Survey of Income and Education. *N: menos de 25 casos.

Reproducido de: Frank D. Bean et al., "Patterns of Fertility Variation Among Mexican Inmigrants", 1980 , pp. 41-42.

En relación con la población de México, la información sobre su comportamiento reciente muestra que también existe una estrecha asociación negativa entre niveles de fecundidad y condiciones económicas y sociales, utilizando la educación como indicador de estas condiciones. La asociación es comparable a la encontrada en los casos de las poblaciones mexicano-americana y blanca norteamericana. De la información contenida en el cuadro 7 se desprende que existe un claro punto de ruptura entre quienes no han completado la educación 
elemental ( 6 años) y quienes sí la han completado: en el primer caso la tasa global de fecundidad es 6.1 o mayor; en el segundo 3.8 o menor.

Cuadro 7

\begin{tabular}{|c|c|c|}
\hline Nivel educativo & & TGF \\
\hline Sin escuela & & 6.7 \\
\hline Primaria incompleta & & 6.1 \\
\hline Primaria completa & & 3.8 \\
\hline Secundaria & & 3.3 \\
\hline Preparatoria y mas & & 2.1 \\
\hline
\end{tabular}

La asociación negativa entre fecundidad y educación se presenta en todos los grupos de edad, como se puede observar en el cuadro 8. Si bien la información disponible sobre fecundidad según nivel educativo entre las distintas poblaciones aquí consideradas no es directamente comparable, las cifras para las mujeres mexicanas de 20 a 34 años de edad (cuadro 8) y las de las mexicano-americanas (cuadro 6) sugieren, sin embargo, que el nivel de fecundidad de las mujeres mexicanas sin escolaridad o con primaria incompleta es más elevado que el de las mexicano-americanas. Se observa, además, que la fecundidad de las mujeres mexicanas (grupo de edades 20-34) con primaria incompleta es 3.2 ó 3.1 veces más elevada que la fecundidad de las mujeres con preparatoria y más escolaridad (datos de 1976 y 1979 respectivamente; ver cuadro 8 ). Entre las mujeres mexicano-americanas la fecundidad de aquellas con los más bajos niveles de escolaridad es sólo 2.7 ó 2.8 veces más alta que la de aquellas con los niveles más elevados (datos de 1970 y 1976 respectivamente; ver cuadro 6).

\section{Resumen y conclusiones}

Los datos que aquí se han presentado son ciertamente muy limitados, como lo es la revisión de la literatura. Sin embargo, ellos permiten derivar tentativamente algunas conclusiones.

Los niveles generales de fecundidad de la población mexicano-americana parecen situarse en el mismo rango que los niveles de la pobla- 
Cuadro 8

Promedio de hijos nacidos vivos por grupos quinquenales de edad y nivel de escolaridad. México, 1976, 1979.

\begin{tabular}{|c|c|c|c|c|c|c|c|c|c|c|}
\hline \multirow{3}{*}{$\begin{array}{l}\text { Grupos } \\
\text { de } \\
\text { edades }\end{array}$} & \multicolumn{10}{|c|}{ Nivel de escolaridad } \\
\hline & \multicolumn{2}{|c|}{ Sin estudios } & \multicolumn{2}{|c|}{$\begin{array}{l}\text { Primaria } \\
\text { incompleta }\end{array}$} & \multicolumn{2}{|c|}{$\begin{array}{l}\text { Primaria } \\
\text { completa }\end{array}$} & \multicolumn{2}{|c|}{ Secundaria } & \multicolumn{2}{|c|}{$\begin{array}{c}\text { Preparatoria } \\
\text { y más }\end{array}$} \\
\hline & 1976 & 1979 & 1976 & 1979 & 1976 & 1979 & 1976 & 1979 & 1976 & 1979 \\
\hline $20-24$ & 2.1 & 2.3 & 1.8 & 1.9 & 1.2 & 1.2 & 0.8 & 0.7 & 0.3 & 0.3 \\
\hline $25-29$ & 4.2 & 4.0 & 3.6 & 3.4 & 2.3 & 2.2 & 1.7 & 1.7 & 1.3 & 1.1 \\
\hline $30-34$ & 5.9 & 5.6 & 5.1 & 4.9 & 3.8 & 3.4 & 2.8 & 2.7 & 1.6 & 1.9 \\
\hline $35-39$ & 7.0 & 6.6 & 6.5 & 6.5 & 4.9 & 4.4 & 3.8 & 3.3 & 3.3 & 2.7 \\
\hline $40-44$ & 7.5 & 7.4 & 7.0 & 7.1 & 5.0 & 4.9 & 4.2 & 3.6 & 3.3 & 3.0 \\
\hline $45-49$ & 7.3 & 7.7 & 7.2 & 7.2 & 6.3 & 5.1 & 3.6 & 3.9 & 3.2 & 4.0 \\
\hline $\begin{array}{l}20-34^{*} \\
\text { (average) }\end{array}$ & e) 4.1 & 4.0 & 3.5 & 3.4 & 2.4 & 2.3 & 1.7 & 1.7 & 1.1 & 1.1 \\
\hline
\end{tabular}

Fuente: Encuesta Mexicana de Fecundidad (1976); Encuesta Nacionāl de Prevalencia en el uso de métodos anticonceptivos con módulo de fecundidad y mortalidad (1979). Datos tomados de: Fecundidad y Uso de Métodos Anticonceptivos en México, IMSS, 1981 , p. $3 \overline{6}$.

* Calculos del autor. Promedio simple de los grupos de edades 20-24, 2529 y $30-34$.

ción mexicana, si bien aquellos son ligeramente inferiores a estos últimos. En ambas poblaciones, la fecundidad varía según condición socioeconómica (escolaridad) de manera similar: los diferenciales de fecundidad según posición social (escolaridad) son bastante amplios y mayores que los que se encuentran en el patrón diferencial de aquellas poblaciones que han completado su transición demográfica. Así, la fecundidad entre los menos educados de la población mexicano-americana está por encima de la de la mayoría de la población norteamericana; mientras que entre la población más educada las diferencias se reducen considerablemente con tendencia a decrecer en el tiempo. En consecuencia, la fecundidad de las otras mujeres blancas norteamericanas con los menores niveles de escolaridad es sólo de 2.2 a 2.4 veces más elevada que la de aquellas con niveles de escolaridad superiores frente un diferencial que se amplía a 2.7 ó 2.8 entre las mujeres mexicano-americanas (ver cuadro 6).

El análisis de la información disponible permite interpretar los diferenciales agregados entre la población mexicano-americana y la población norteamericana en función de las diferencias agregadas entre estas poblaciones en las características socioeconómicas respectivas; el número de mujeres mexicano-americanas con elevados niveles de escolaridad es muy reducido comparado con el resto de la población. Sin embargo, aún en el caso de incorporar al análisis varia- 
bles de control, como la escolaridad, las diferencias en fecundidad de las mujeres mexicano-americanas se mantiene a todos los niveles de escolaridad, con excepción del más elevado (ver cuadro 6). Lo anterior no permite descartar que la fecundidad entre la población mexicano-americana no se asemejará a la de otros grupos norteamericanos al incrementarse su nivel educativo.

Los patrones observados - los diferenciales en fecundidad entre la población norteamericana de origen mexicano y la población blanca mayoritaria se reducen al controlar por condición sócioeconómica, pero no se eliminan del todo- sugieren que los factores socioeconómicos y los factores culturales interactúan en la conformación del resultado final del comportamiento de la fecundidad. En el mismo sentido se puede interpretar el hallazgo de que los diferenciales en fecundidad entre las dos poblaciones se reducen conforme las generaciones de mexicano-americanos son más antiguas, pero las diferencias persisten. Ambos conjuntos de factores -integración socioeconómica y permanencia en los Estados Unidos- influyen en el comportamiento de la fecundidad de la población de origen mexicano en dicho país. Sin embargo, la adopción de un patrón de fecundidad reducida por parte de esta población parecería más dependiente de su integración estructural a la economía y sociedad norteamericanas -educaciónque de cualquier otro factor. Como lo indica un estudio reciente, es más probable que las mujeres mexicano-americanas con altos niveles de educación reporten niveles de fecundidad similares a los del grupo (restante) de mujeres blancas que lo reporten las mujeres de generaciones con residencia más prolongada. ${ }^{12}$

Los análisis que se han realizado hasta el presente sobre la población de origen mexicano en los Estados Unidos no dan respuesta cabal a la cuestión de si los diferenciales en el comportamiento de la fecundidad entre las poblaciones mexicano-americanas y norteamericana, $o$ mexicana, pueden ser claramente atribuibles a diferencias culturales o a diferencias socioeconómicas. La única consideración concluyente que de dichos estudios podría derivarse, usando las palabras de uno de los estudios, es que "ethnicity is not a bag of norms producing automatic responses... ethnicity es variable and relative." 13 En otras palabras, y en un sentido que reclama esfuerzos adicionales, la incorporación al análisis del comportamiento reproductivo de características y condiciones socioeconómicas y de los sistemas normativos que se heredan históricamente exige el uso de estructuras conceptua-

12 Frank D. Bean et al., "Patterns of Fertility Variation Among Mexican Immigants", 1980, p. 52.

13 David E. Lopez y George Sabagh, "Untangling Struchural and Normative Aspects of the Minority Status Fertility Hypothesis", American Joumal of Sociology, 1978, p. 1496. 
les complejas capaces de reflejar los arreglos institucionales esenciales que pudieran esclarecer las vinculaciones entre los códigos normativos y culturales y los sistemas de control económico y social sobre la fecundidad. ${ }^{14}$

${ }^{14}$ Véase Ron Lesthaeghe, "On the Social Control of Human Reproduction", Population and Development Review, december, 1980, pp. 527-548. 